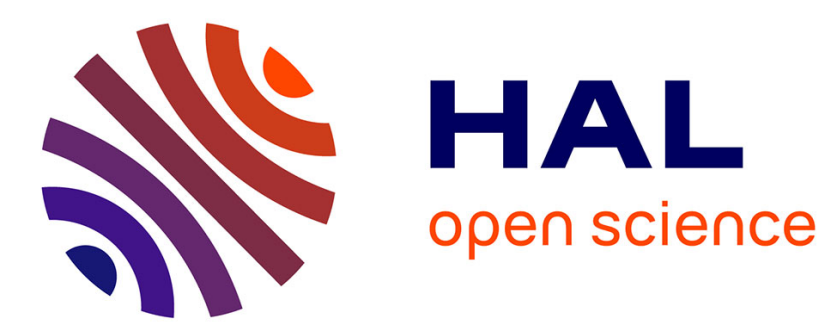

\title{
Low complexity iterative receiver for Non-Orthogonal Space-Time Block Code with channel coding
}

Pierre-Jean Bouvet, Maryline Hélard, Vincent Le Nir

\section{To cite this version:}

Pierre-Jean Bouvet, Maryline Hélard, Vincent Le Nir. Low complexity iterative receiver for Non-

Orthogonal Space-Time Block Code with channel coding. 2004, 5 p. hal-00005813

\section{HAL Id: hal-00005813 \\ https://hal.science/hal-00005813}

Submitted on 4 Jul 2005

HAL is a multi-disciplinary open access archive for the deposit and dissemination of scientific research documents, whether they are published or not. The documents may come from teaching and research institutions in France or abroad, or from public or private research centers.
L'archive ouverte pluridisciplinaire HAL, est destinée au dépôt et à la diffusion de documents scientifiques de niveau recherche, publiés ou non, émanant des établissements d'enseignement et de recherche français ou étrangers, des laboratoires publics ou privés. 


\title{
Low complexity iterative receiver for Non-Orthogonal Space-Time Block Code with channel coding
}

\author{
Pierre-Jean Bouvet, Maryline Hélard, Member, IEEE, Vincent Le Nir \\ France Telecom R\&D \\ 4 rue du Clos Courtel 35512 Césson-Sévigné France \\ Email: pierrejean.bouvet@rd.francetelecom.com
}

\begin{abstract}
In this paper, we study the association of Nonorthogonal Space-Time Block Coding (NO-STBC) with channel coding and propose an efficient iterative receiver. For two transmit antennas, the use of the Alamouti scheme as STBC is optimal owing to its orthogonality and its unitary rate. For more than two antennas, full rate is maintained by giving up the orthogonality. This non-orthogonality makes the decoding often complex and sub-optimal in terms of exploitation of the diversity. In this paper, we present a low complexity receiver based on iterative process performing jointly space-time decoding and channel decoding. Simulations over uncorrelated Rayleigh flat fading channels show that such receiver exploits at best the space-time diversity provided by the Non-Orthogonal STBC and the channel coding.
\end{abstract}

\section{INTRODUCTION}

In wireless communications systems, the fading effects of the channel significantly degrade the performance. This phenomena can be efficiently combated by the use of transmit diversity techniques. Since 1998 and the pioneering work of Tarokh and Alamouti [1][2], space-time diversity techniques have been studied extensively. An attractive approach to exploit such diversity is Space-Time Block Coding (STBC). When taken from orthogonal design, these codes provide full diversity and allow a simple decoding algorithm, we talk about Orthogonal Space Time Block Coding (O-STBC) [2][3]. However it has been proved in [3] that, for complex modulation, there are no full rate O-STBC for more than 2 Tx antennas and their extension to a greater number of antennas is only possible with lower transmission rate $(1 / 2$ or $3 / 4$ for example). One major technique to overcome the rate limitation of O-STBC was proposed in [4][5] by introducing full rate Non-Orthogonal STBC (NO-STBC). Because of the non-orthogonality of the structure, a classical Maximum Ratio Combining (MRC) performed on the receiver side to carry out STBC decoding brings Inter Element Interference (IEI) thus requiring an Maximum Likelihood (ML) algorithm [4]. Nevertheless, reduced complexity receiver based on linear algorithms are presented in [5][6]. On the other hand, the con- catenation of channel coding and O-STBC has been studied in [7]: the best performance versus complexity trade-off is demonstrated to be achieved by choosing the Alamouti OSTBC as space-time code [2]. In this paper, we consider the combination of channel coding with NO-STBC and propose an iterative receiver which takes benefit from the channel decoding in order to improve the space-time block decoding by removing the interference terms. Simulations show that such receiver exploits the diversity in an optimal manner while keeping low complexity.

\section{SYSTEM MODEL AND NOTATIONS}

\section{A. Transmitter}

Information bits $d_{n}$ are first channel encoded and then interleaved (П). The output bits are passed directly to a mapper leading to complex modulated symbols $s_{k}$. We consider a $M$-state modulation. Each group of $K$ symbols are spacetime encoded employing a space-time block encoder and then transmitted over $T$ symbol durations. Assuming $N_{t}$ transmit antennas, the output of the STBC is a $T \times N_{t}$ matrix $\mathbf{C}=\left[c_{p i}\right]$, where $c_{p i}$ is either $\pm s_{k}$ or $\pm s_{k}^{*}$ and is transmitted by antenna $i$ at time $t+(p-1) T_{s}$. Superscript $(.)^{*}$ denotes complex conjugaison operation. The rate of the space-time code is defined to be $R=K / T$. The STBC is said to be orthogonal if:

$$
\mathbf{C}^{H} \mathbf{C}=\left(\left|s_{1}\right|^{2}+\cdots+\left|s_{k}\right|^{2}\right) \mathbf{I}
$$

Where $\mathbf{I}$ is the $N_{t} \times N_{t}$ identity matrix.

\section{B. Channel model}

We consider a discret-time MIMO channel model with $N_{t}$ transmit antennas and $N_{r}$ receive antennas as described in Figure 1. Each sub-channels are supposed independent meaning that antenna spacing is sufficient. Frequency and time selective channels is considered. By using Orthogonal Frequency Division Multiplex (OFDM) modulation and demodulation, a frequency selective channel is transformed into 
a set of orthogonal flat Rayleigh fading sub-channels [8]. Finally, the channel is supposed to be constant over $T$ symbol durations. Thus, the channel coefficient from antenna $i$ to the receive antenna $j$ can be modeled by a complex value $h_{i j}$. Assuming ideal interleaving in the OFDM process, the $h_{i j}$ 's samples follow an uncorrelated complex Gaussian law with unit variance. At time $t+(p-1) T_{s}$, the received signal $r_{p, j}$ at antenna $j$ is given by:

$$
r_{p, j}=\sum_{i=1}^{N_{t}} h_{i, j} c_{p i}+n_{p, j}
$$

where the noise samples $n_{j, p}$ are independent samples of a zero-mean complex Gaussian random variable with a total variance of $\sigma_{n}^{2}$. Effects of both channel and space time coding can be represented by an equivalent channel matrix $\mathbf{H} \in$ $\mathbb{C}^{N_{r} T \times K}$ [6] [9]. By introducing an equivalent receive vector $\mathbf{r} \in \mathbb{C}^{N_{r} T \times 1}$ whose components are either $r_{p, j}$ or $r_{p, j}^{*}$, we can write (see section $\mathrm{V}$ ):

$$
\mathbf{r}=\mathbf{H s}+\mathbf{n}
$$

where $\mathbf{s}=\left[\begin{array}{lll}s_{1} & \ldots & s_{K}\end{array}\right]^{T}$ and $\mathbf{n} \in \mathbb{C}^{N_{r} T \times 1}$ is the equivalent noise vector. The average power of the symbols transmit from the $N_{t}$ antennas is normalized to be 1 so that the average power at each receive antenna is 1 and the signal-to-noise ratio (SNR) per antenna is equal to $1 / \sigma_{n}^{2}$. We assume that perfect channel information is available on the receiver side. This can be accomplished by classical means of channel estimation, e.g. insertion of pilot symbols or pilot tones.

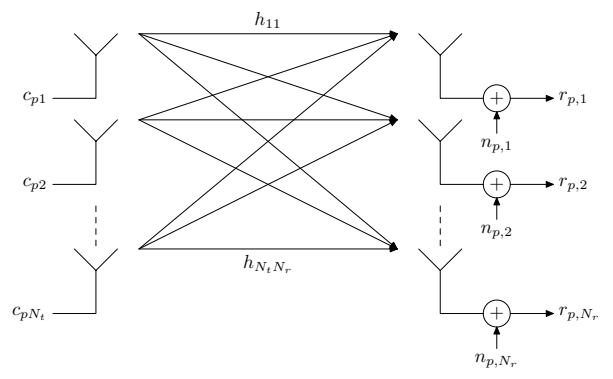

Fig. 1. MIMO Channel model

\section{STBC Decoding strategies}

Classical decoding strategy for STBC is MRC. This decoding is performed by applying $\mathbf{H}^{H}$ to equivalent receive vector r:

$$
\hat{\mathbf{s}}_{M R C}=\mathbf{H}^{H} \mathbf{r}=\mathbf{H}^{H} \mathbf{H} \mathbf{s}+\mathbf{H}^{H} \mathbf{n}
$$

For orthogonal STBC, it can be shown that equivalent matrix $\mathbf{H}$ has the same orthogonality properties as matrix $\mathbf{C}$. Under this assumption eq (4) becomes:

$$
\hat{\mathbf{s}}_{M R C}=\mathbf{H}^{H} \mathbf{r}=\sum_{i} \sum_{j}\left|h_{i j}\right|^{2} \mathbf{s}+\mathbf{H}^{H} \mathbf{n}
$$

Thus orthogonality ensures linear decoding and optimal diversity exploitation. MRC decoding leads to the same performance as a Maximum Likelihood decoder [10].

In case of Non orthogonal STBC, matrix $\mathbf{H}$ is no more orthogonal and equation (4) becomes:

$$
\hat{\mathbf{s}}_{M R C}=\sum_{i} \sum_{j}\left|h_{i j}\right|^{2} \mathbf{s}+\mathbf{J} \mathbf{s}+\mathbf{n}
$$

where $\mathbf{J}=\mathbf{H}^{H} \mathbf{H}-\operatorname{diag}\left(\mathbf{H}^{H} \mathbf{H}\right)$ with $\mathbf{J} \in \mathbb{C}^{K \times K}$. The estimated vector $\hat{\mathbf{s}}_{M R C}$ is corrupted by Inter Element Interference (IEI) represented by $\mathbf{J} \mathbf{s}$. These interferer degrade severely the performance and prevent from linear decoding [4].

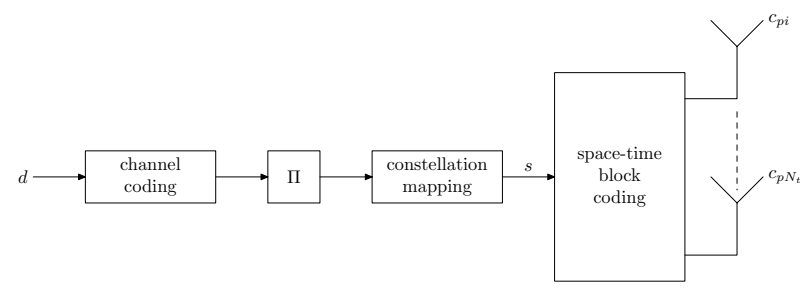

Fig. 2. Transmitter

\section{ITERATIVE SPACE-TIME DECODING AND CHANNEL DECODING RECEIVER}

In case of O-STBC, optimal performance are obtained by simply concatenating space-time and channel decoding in the receiver [7]. In case of NO-STBC, because of the nonorthogonality, the optimal receiver would consist in performing jointly space-time decoding and channel decoding owing to a super trellis. However this receiver is extremely complex and could not reasonably be implemented in a ship. In this paper we propose a practical sub-optimum decoding scheme based on iterative "turbo" principle [11] where two stages, a space-time demapper and a channel decoder exchange extrinsic information in an iterative loop until the receiver converges. These stages are separated by an interleaver used to decorrelate the outputs before feeding them to the next decoding stage. Figure 3 describes the proposed scheme. The space-time decoder employs a linear Parallel Soft Interference Canceller (PSIC) optimized under the Minimum Mean-Square Error (MMSE) criterion which aim is to restore the orthogonality by removing the IEI. For the first iteration, because no prior information on transmitted symbols is available, the decoded vector $\tilde{\mathbf{s}}^{(1)}$ is obtained by a linear MMSE space-time decoder:

$$
\tilde{\mathbf{s}}^{(1)}=\left(\mathbf{H}^{H} \mathbf{H}+\sigma_{n}^{2} \mathbf{I}\right)^{-1} \mathbf{H}^{H} \mathbf{r}
$$

For next iterations $(l>1)$, previous estimated symbols are used by the PSIC decoder in order to cancel the residual IEI:

$$
\tilde{\mathbf{s}}^{(l)}=\left(\operatorname{diag}\left(\mathbf{H}^{H} \mathbf{H}\right)+\sigma_{n}^{2} \mathbf{I}\right)^{-1}\left(\mathbf{H}^{H} \mathbf{r}-\mathbf{J} \hat{\mathbf{s}}^{(l-1)}\right)
$$


where $\mathbf{J} \in \mathbb{C}^{K \times K}$ is the IEI matrix: $\mathbf{J}=\mathbf{H}^{H} \mathbf{H}-\operatorname{diag}\left(\mathbf{H}^{H} \mathbf{H}\right)$ and $\mathbf{I}$ is the $K \times K$ identity matrix.

The second stage is a channel decoder based on Soft Output Viterbi Output (SOVA) algorithm which produces extrinsic Logarithmic Likelihood Ratios (LLRs) on coded bits that are fed to the first stage. By performing successively and

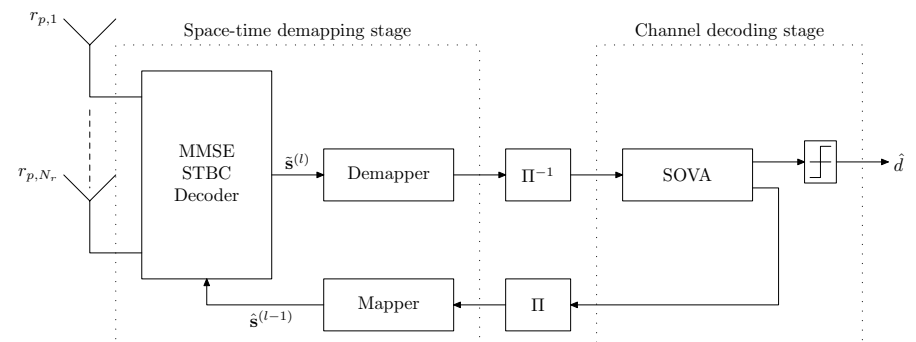

Fig. 3. Proposed iterative Receiver

iteratively channel decoding and interferer terms cancelling, global STBC decoding is progressively improved

\section{STUdied SYSTEMS}

We consider three different MIMO systems for which no full rate full diversity O-STBC exist.

\section{A. $4 \times 1$ system with rate 1 Quasi-orthogonal STBC}

The rate-one Jafarkhani code is used [4]:

$$
\mathbf{C}_{\text {Jaf } 4}=\left[\begin{array}{cccc}
s_{1} & s_{2} & s_{3} & s_{4} \\
-s_{2}^{*} & -s_{1}^{*} & -s_{4}^{*} & s_{3}^{*} \\
-s_{3}^{*} & s_{4}^{*} & s_{1}^{*} & s_{2}^{*} \\
s_{4} & -s_{3} & -s_{2} & s_{1}
\end{array}\right]
$$

By using the following equivalent channel matrix:

$$
\mathbf{H}=\left[\begin{array}{cccc}
h_{11} & h_{21} & h_{31} & h_{41} \\
h_{21}^{*} & -h_{11}^{*} & h_{41}^{*} & -h_{31}^{*} \\
h_{31}^{*} & h_{41}^{*} & -h_{11}^{*} & -h_{21}^{*} \\
h_{41} & -h_{31} & -h_{21} & h_{11}
\end{array}\right]
$$

and the following equivalent receive vector:

$$
\mathbf{r}=\left[\begin{array}{llll}
r_{1,1} & r_{2,1}^{*} & r_{3,1}^{*} & r_{4,1}
\end{array}\right]^{T}
$$

we obtain this interference matrix:

$$
\mathbf{J}=\left[\begin{array}{cccc}
0 & 0 & 0 & a \\
0 & 0 & -a & 0 \\
0 & -a & 0 & 0 \\
a & 0 & 0 & 0
\end{array}\right]
$$

with $a=h_{1} h_{4}^{*}-h_{2} h_{3}^{*}-h_{3} h_{2}^{*}+h_{4} h_{1}^{*}$. Because there are low interference terms, the Jafarkhani code is said quasiorthogonal.

\section{B. $3 \times 1$ system with rate $1 \mathrm{NO}-\mathrm{STBC}$}

For this transmission scheme, a repetition based STBC is used:

$$
\mathbf{C}_{3}=\left[\begin{array}{lll}
s_{1} & s_{2} & s_{3} \\
s_{3} & s_{1} & s_{2} \\
s_{2} & s_{3} & s_{1}
\end{array}\right]
$$

This code leads to the following equivalent channel matrix:

$$
\mathbf{H}=\left[\begin{array}{lll}
h_{11} & h_{21} & h_{31} \\
h_{31} & h_{11} & h_{21} \\
h_{21} & h_{31} & h_{11}
\end{array}\right]
$$

and the following receive vector:

$$
\mathbf{r}=\left[\begin{array}{lll}
r_{1} & r_{2} & r_{3}
\end{array}\right]^{T}
$$

The STBC is full-rate $(R=1)$ and the interference matrix $J$ shows that the STBC is not orthogonal:

$$
\mathbf{J}=\left[\begin{array}{ccc}
0 & a & b^{*} \\
b^{*} & 0 & b \\
b & b^{*} & 0
\end{array}\right]
$$

with $b=h_{2} h_{1}^{*}+h_{1} h_{3}^{*}+h_{3} h_{2}^{*}$

\section{C. $4 \times 2$ system with rate $2 \mathrm{NO}-\mathrm{STBC}$}

For future wireless communications, high data rates will have to be provided. In order to reach these high data rates, spatial diversity and spatial multiplexing must be efficiently used as studied within the framework of the IST-4MORE project where $4 \times 2$ systems based on MC-CDMA are under study in a downlink scenario [12]. For this MIMO architecture, we propose to use a NO-STBC combining spatial multiplexing and O-STBC initially introduced by Bäro et al. in [13]. Two Alamouti codes are performed simultaneously on two blocks of two antennas leading to a code rate of 2 :

$$
\mathbf{C}_{\text {Bäro }}=\left[\begin{array}{cccc}
s_{1} & s_{2} & s_{3} & s_{4} \\
-s_{2}^{*} & s_{1}^{*} & -s_{4}^{*} & s_{3}^{*}
\end{array}\right]
$$

Note that this transmission scheme belongs to the Linear Dispersion Codes category formalized by Hassibi and Hochwald [14]. By using the following transmission matrix:

$$
\mathbf{H}=\left[\begin{array}{cccc}
h_{11} & h_{21} & h_{31} & h_{41} \\
h_{12} & h_{22} & h_{32} & h_{42} \\
h_{21}^{*} & -h_{11}^{*} & h_{41}^{*} & -h_{31}^{*} \\
h_{22}^{*} & -h_{12}^{*} & h_{42}^{*} & -h_{32}^{*}
\end{array}\right]
$$

and the following equivalent receive vector:

$$
\mathbf{r}=\left[\begin{array}{llll}
r_{1,1} & r_{2,1}^{*} & r_{1,2} & r_{2,2}^{*}
\end{array}\right]^{T}
$$

The resulting interference matrix is the following:

$$
\mathbf{H}=\left[\begin{array}{cccc}
0 & 0 & u & v \\
0 & 0 & -v^{*} & u^{*} \\
u^{*} & -v & 0 & 0 \\
v^{*} & u & 0 & 0
\end{array}\right]
$$


with $u=h_{31} h_{11}^{*}+h_{21} h_{41}^{*}+h_{32} h_{12}^{*}+h_{22} h_{42}^{*}$ and $v=$ $-h_{21} h_{31}^{*}+h_{41} h_{11}^{*}-h_{22} h_{32}^{*}+h_{42} h_{12}^{*}$

\section{Simulation Results}

Considering the $R_{c}=1 / 2$ convolutional encoder with polynomial generator $(133,177)_{o}$ and a QPSK modulation, we provide simulation results obtained with the proposed iterative receiver for the three different NO-STBC systems presented in the previous section. Curves are plotted in signal-to-noise ratio per useful bit $E_{b} / N_{0}$. The relation with SNR is the following:

$$
E_{b} / N_{0}=\left(\log _{2}(M) R_{c} R\right)^{-1} S N R
$$

For each system, we provide performance of the first iteration (\#1) and performance of the iteration for which the process has converged. All performance results are compared to an optimum curve defined as the lower bound which represents a genie aided space-time decoder cancelling perfectly all the IEI.

\section{A. $4 \times 1$ system with rate 1 Quasi-orthogonal STBC}

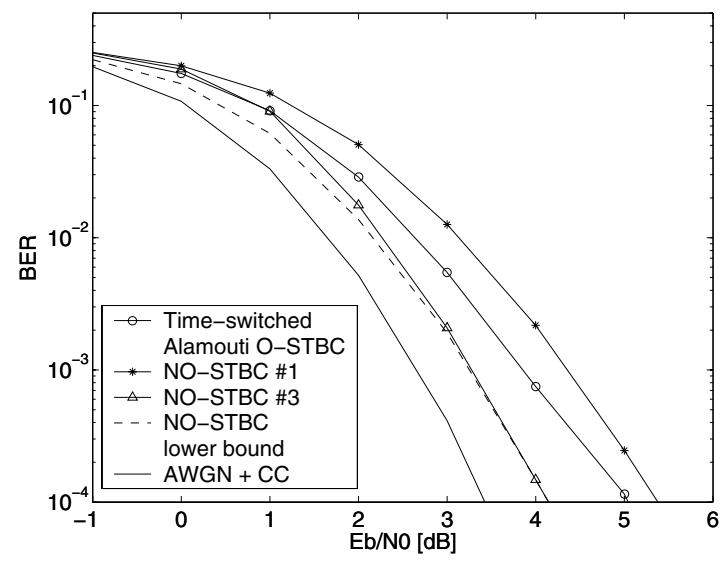

Fig. 4. Performance of the channel coded Jafarkhani $4 \times 1$ NO-STBC scheme with the proposed iterative receiver over uncorrelated Rayleigh flat fading channels, $1 \mathrm{bit} / \mathrm{s} / \mathrm{Hz}$

Figure 4 provides performance results of $4 \times 1$ system with the Jafarkhani STBC and the proposed iterative receiver. For comparison, we also show the performance of so called 4antennas time-switched Alamouti O-STBC combined with the same channel encoder. The coding matrix is the following [15]:

$$
\mathbf{C}_{A l 4}=\left[\begin{array}{cccc}
s_{1} & s_{2} & 0 & 0 \\
-s_{2}^{*} & s_{1}^{*} & 0 & 0 \\
0 & 0 & s_{3} & s_{4} \\
0 & 0 & -s_{4}^{*} & s_{3}^{*}
\end{array}\right]
$$

The STBC is unitary rate and provides a 2-order spatial diversity. The receiver only consists of a STBC decoder concatenated with the channel decoder. Without iterative process, we can notice that the performance of the 4-antenna Jafarkhani code is worse than the 4-antenna time-switched Alamouti code. This is due to the IEI brought by the quasi-orthogonal Jafarkhani code. However the IEI is progressively removed by the iterative process, and after only 3 iterations the lower bound performance is reached at high SNR meaning that all the interference terms brought by the NO-STBC are completely removed. In fact, the lower bound curve corresponds to a virtual 4-antenna O-STBC in conjunction with channel coding and represents the maximum achievable diversity for such a system. Thus the iterative process efficiently exploits the 4-order diversity brought by the Jafarkhani code leading to a significant performance gain over the 4-antennas time-switched Alamouti system. At low SNR, the process does not succeed in removing all the IEI leading to a degradation versus the optimum curve. The quasi-orthogonality properties of the Jafarkhani code explained that this degradation is relatively small. Full diversity Tarokh O-STBC [10] could be used for this system, but due to their lower rate (1/2 or $3 / 4)$, these codes ought to be associated with higher order modulation and thus limiting their performance.

\section{B. $3 \times 1$ system with rate $1 \mathrm{NO}-\mathrm{STBC}$}

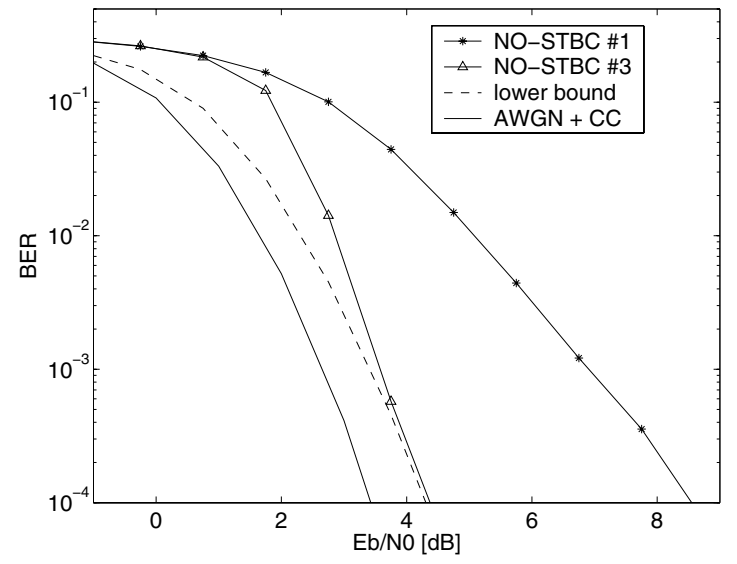

Fig. 5. Performance of the channel coded $3 \times 1$ NO-STBC scheme with the proposed iterative receiver over uncorrelated Rayleigh flat fading channels, 1 $\mathrm{bit} / \mathrm{s} / \mathrm{Hz}$

After having tested the efficiency of the proposed receiver for a quasi-orthogonal STBC, the receiver is now evaluated for a non-orthogonal STBC in a $3 \times 1$ system which performance results are plotted in Figure 5. Due to higher interference terms, the $3 \times 1$ system provides, at the first iteration, worse performance than the $4 \times 1$ Jafarkhani system. However, with the same convergence speed (3 iterations), lower bound is reached leading to a $4 \mathrm{~dB}$ gain over the first 
iteration. One can notice that the degradation at low SNR is much significant than for the Jafarkhani system. This can also be explained by the several interference terms brought the 3 -antennas NO-STBC. Our system could also be compared with the rate 3/4 O-STBC for 3 antennas presented in [10]. However it well known, that the STBC rate must be much favoured than modulation order.

\section{C. $4 \times 2$ system with rate 2 NO-STBC}

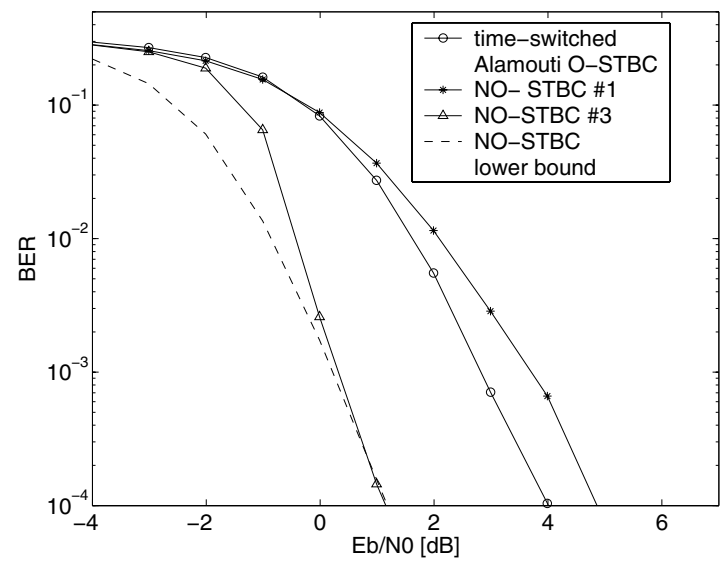

Fig. 6. Performance of the channel coded $4 \times 2$ NO-STBC scheme with the proposed iterative receiver over uncorrelated Rayleigh flat fading channels, 2 $\mathrm{bit} / \mathrm{s} / \mathrm{Hz}$

Figure 6 shows the performance of the $4 \times 2$ system. The rate-2 NO-STBC leads to spectral efficiency of $2 \mathrm{bits} / \mathrm{s} / \mathrm{Hz}$. As shown in Figure 5, the presence of several terms in the interference matrix, leads to lack in performance at low SNR. However optimal performance are also reached from $E_{b} / N_{0}=$ $0 \mathrm{~dB}$. For comparison, we also consider the 4-antennas timeswitched Alamouti STBC $\left(\mathbf{C}_{A l 4}\right)$ with a 16-QAM modulation (leading to the same spectral efficiency i.e. 2 bits $/ \mathrm{s} / \mathrm{Hz}$ ). Simulation results show that the orthogonality of the 4-antenna time-switched Alamouti code does not compensate the lack in STBC rate.

\section{CONCLUSION}

In this paper, we investigate NO-STBC in conjunction with channel coding in order to both exploit the channel coding and the space-time diversities. We propose an efficient iterative receiver leading to a good trade-off between complexity and performance. With a very small number of iterations the process converges to the maximum diversity curve. Because full rate and full diversity O-STBC only exist for 2 transmit antenna schemes, channel coded NO-STBC associated with the proposed receiver seems to be suitable for exploiting space-time diversity in an optimal way without sacrificing data rate and whatever the number of transmit or receive antennas.

\section{ACKNOWLEDGMENT}

Part of this work has been carried out whithin the framework of the IST 4MORE project [12].

\section{REFERENCES}

[1] V. Tarokh, N. Seshadri, and R. Calderbank, "Space-time codes for high data rate wireless communication: performance criterion and code construction," IEEE Trans. Inform. Theory, vol. 44, no. 2, pp. 744-765, Mar. 1998.

[2] S. M. Alamouti, "A simple transmit diversity technique for wireless communications," IEEE J. Select. Areas Commun., vol. 16, no. 8, pp. 1451-1458, Oct. 1998.

[3] V. Tarokh, H. Jafarkhani, and R. Calderbank, "Space-time block codes from orthogonal designs," IEEE Trans. Inform. Theory, vol. 45, no. 4, pp. 1456-1467, July 1999.

[4] H. Jafarkhani, "A quasi-orthogonal space-time block code," IEEE Trans. Commun., vol. 49, no. 1, pp. 42-45, Jan. 2001.

[5] O. Tirkkonen, A. Boariu, and A. Hottinen, "Minimal non-orthogonality rate 1 space-time block code for 3+ tx antennas," in Proceedings of ISSSTA'00, New jersey, USA, Sept. 2000, pp. 429-432.

[6] A. Boariu and D. M. Ionescu, "A class of nonorthogonal rate-one spacetime block codes with controlled interference," IEEE Trans. Commun., vol. 2 , no. 2 , pp. 270-276, Mar. 2003.

[7] T. H. Liew and L. Hanzo, "Space-time codes and concatenated channel codes for wireless communications," Proceedings of the IEEE, vol. 90, no. 2, pp. 187-219, Feb. 2002.

[8] B. Le Floch, M. Alard, and C. Berrou, "Coded orthogonal frequency division multiplex," Proceedings of the IEEE, vol. 83, no. 6, pp. 982996, June 1995.

[9] D. Gesbert, M. Shafi, D. Shiu, P. Smith, and A. Naguib, "From theory to practice: an overview of MIMO space-time coded wireless systems," IEEE J. Select. Areas Commun., vol. 21, no. 3, pp. 281-301, Apr. 2003.

[10] V. Tarokh, H. Jafarkhani, and A. R. Calderbank, "Space-time block coding for wireless communications: Performance results," IEEE J. Select. Areas Commun., vol. 17, no. 3, pp. 451-460, Mar. 1999.

[11] C. Laot, A. Glavieux, and J. Labat, "Turbo equalization: adaptive equalization and channel decoding jointly optimized," IEEE J. Select. Areas Commun., vol. 19, no. 9, pp. 1744-1752, Sept. 2001.

[12] "IST 4MORE project," http://www.ist-4more.org.

[13] S. Baro, G. Bauch, A. Pavlic, and A. Semmler, "Improving BLAST performance using space-time block codes and turbo decoding," in Proceedings of GLOBECOM'00, San Francisco, USA, Nov. 1998, pp. 1067-1071.

[14] B. Hassibi and B. M. Hochwald, "High-rate codes that are linear in space and time," IEEE Trans. Inform. Theory, vol. 48, no. 7, pp. 18041824, July 2002.

[15] M. Ivrlac and W. Utschick, "On time-switched space-time transmit diversity in MISO systems," in Proceedings of VTC Fall '02, Vancouver, Canada, Sept. 2002. 